\title{
Control de proyectos de Responsabilidad Social empresarial: Estudio en empresas mineras
}

\author{
Rojas de la Puente, Edward Enrique ${ }^{1}$ \\ Fanny Lucila Rimarachín Chavez ${ }^{2}$ \\ Oblitas Cruz, Jimy ${ }^{3}$ \\ Castro Silupu, Wilson ${ }^{4}$
}

\section{Resumen}

El objetivo de esta investigación es analizar el control de proyectos de responsabilidad social empresarial en el sector minero con la finalidad de proponer indicadores indicadores relevantes de responsabilidad social ajustado a las necesidades de los proyectos desarrollados por dichas empresas. La metodología se dividió en 1) Identificación de los principales indicadores de Responsabilidad Social en proyectos mineros; 2) Revisión por expertos de los índices de responsabilidad identificando criterios relevantes en minería; y 3) Identificación y diseño de los indicadores de Responsabilidad Social Empresarial.

Recibido: 20.02 .19 Aceptado: 20.06 .19

$1 \quad$ Licenciado en Lengua y Literatura. Mg. En Lectura y Escritura (Universidad Nacional de Carabobo -Valencia - Venezuela). Dr. En Administración de la educación. Con estudios de Sociología y Filosofía en el Seminario Santo Toribio de Mogrovejo y la Pontificia Universidad Católica de Lima. Docente universitario de amplia experiencia. https://orcid.org/0000-0001-9026-1244

2 Ingeniero en Industrias Alimentarias, con Maestría en Gestión Ambiental, especialista en Mejora de Procesos y Gestión de Calidad. Actualmente docente en la Escuela de Industrias Alimentarias de la universidad Nacional de Cajamarca. https://orcid.org/0000-0002-8487-9897

3 lingeniero agroindustrial y recibió la maestría en ciencias y una especialización en administración de operaciones. Actualmente está cursando el Ph.D. Licenciado en la Universidad de Lleida, España. Cuenta con experiencia ejecutiva en la planificación y desarrollo de proyectos, gestionando el Centro de Investigación e Innovaciones para la Agroindustria Peruana. https://orcid.org/0000-0001-7652-6672

$4 \quad$ Licenciado en Ciencias e Ingeniería de la Alimentación y en el Ph.D. Licenciado en ciencia, tecnología y gestión de alimentos por la Universitat Politécnica de Valencia, España. Actualmente es profesor investigador senior en el Departamento de Ingeniería Industrial, Universidad Privada del Norte, Perú. https://orcid. org/0000-0001-7286-1262 
En la primera etapa se analizaron ocho proyectos de Responsabilidad Social Empresarial con financiamiento minero de US \$ 6000 000.00. En la segunda etapa se identificaron diez criterios usados en proyectos mineros. Finalmente, se seleccionaron cinco criterios prioritarios: inversión social, imagen y confianza, prioridades de los proyectos, apalancamiento de recursos - aliados y rentabilidad social, diseñándose siete indicadores. Los resultados indican que las empresas mineras definen y utilizan indicadores concentrados en el desarrollo de las actividades y avance de metas de cada proyecto (operativos o de resultados esperados), sin establecer mediciones de conceptos de impacto social. El uso de indicadores relevantes y específicos en el ámbito social sirve de herramienta y contribuye a generar rentabilidad social para las empresas financiadoras mineras, permitiendo un más efectivo control de proyectos de responsabilidad social, reducir costos y proporcionando valor a largo plazo, razón por la cual se propone un sistema de indicadores para este tipo de empresas.

Palabras clave: responsabilidad social empresarial; indicadores; minería; control de proyectos; criterios de control.

\title{
Control of Corporate Social Responsibility projects: Study in mining companies
}

\begin{abstract}
The objective of this research is to analyze the control of corporate social responsibility projects in the mining sector in order to propose relevant indicators of social responsibility adjusted to the needs of the projects developed by these companies. The methodology was divided into 1) Identification of the main Social Responsibility indicators in mining projects; 2) Expert review of responsibility indices identifying relevant criteria in mining; and 3) Identification and design of the indicators of Corporate Social Responsibility. In the first stage, eight Corporate Social Responsibility projects with mining financing of US $\$ 6,000,000.00$ were analyzed. In the second stage, ten criteria used in mining projects were identified. Finally, five priority criteria were selected: social investment, image and trust, project priorities, resource leverage - allies and social profitability, with seven indicators being designed. The results indicate that mining companies define and use indicators focused on the development of the activities and progress of goals of each project (operational or expected results), without establishing measures of social impact concepts. The use of relevant and specific indicators in the social field serves as a tool and contributes to generating social profitability for mining finance companies, allowing more effective control of social responsibility projects, reducing costs and providing longterm value, which is why a system of indicators is proposed for this type of companies
\end{abstract}

Keywords: Corporate Social Responsibility; indicators; mining; Project Controls; Criteria of Control. 
Control de proyectos de Responsabilidad Social empresarial: Estudio en empresas mineras

Rojas de la Puente, Edward Enrique; Fanny Lucila Rimarachín Chavez; Oblitas Cruz, Jimy y Castro Silupu, Wilson

\section{Introducción}

La responsabilidad social empresarial (RSE) ha tenido una historia difícil en la industria minera y mucho más a nivel internacional donde este tipo de industria se ha movido para reorientarse a través de una serie de iniciativas globales que elaboran formas en que la minería puede y contribuye al desarrollo sostenible (Frederiksen, 2018). La minería es fundamental para los debates sobre el papel de la RSE en la promoción del desarrollo con la RSE en el sector extractivo, el grado en que realmente implementan los principios de la RSE en sus operaciones en el terreno es cuestionable, especialmente en los países en desarrollo (Slack, 2012).

Actualmente la minería tiene como concepto que, "la responsabilidad social debe generar algún tipo de retorno para resultar sostenible, es decir, debe contribuir a la sostenibilidad empresarial de la empresa" (Hoyos y Verónica, 2012), este concepto ha generado muchas veces conflictos como los generados en la empresa Newmont Mining Corporación que en el mismo periodo de tiempo presentó conflictos en Perú, dando como resultado el cierre del proyecto Conga (proyecto por 5000 millones de dólares); y, en una operación similar, en Ghana, contaron con el apoyo total de la comunidad, lo cual ha generado que las corporaciones mineras multinacionales tomen sus responsabilidades sociales más seriamente en las comunidades que operan.

Por otro lado, la relación entre la responsabilidad social empresarial y los indicadores económicos, ha sido objeto de una amplia investigación empírica; sin embargo, aún no se tiene certeza de este tipo de relación ya que muchos de los resultados son diversos y contradictorios, básicamente por la manera de medirlos (Galant y Cadez, 2017). Una gran cantidad de estudios académicos se han dedicado a la investigación de las responsabilidad social empresarial ( $\mathrm{He}$ et al. 2019; Tuulentie et al. 2019; Walker y Sarkodie 2019), y aunque el mundo de los negocios parece haber aceptado la idea general de que debería ser socialmente responsable, existe poca información referente a cómo las empresas perciben estas actividades (Combs et al, 2013).

Es justamente, ante la necesidad de implementar proyectos de responsabilidad social, articulados a las necesidades de las comunidades aledañas a los proyectos mineros que, además cumplan las expectativas de los grupos de interés de las empresas, es que se hace prioritario mejorar la percepción del retorno social que pueden brindar los proyectos, de tal manera que mejore la inversión social privada desde el enfoque de responsabilidad social empresarial (Amos, 2018).

En el actual contexto de las regiones mineras del país, caracterizado por el devenir de conflictos sociales a diferente escala, como los mencionados por diferentes estudios (Dunlap, 2019; Morales et al, 2018; Sícoli y Sallan, 2019) donde en común se observa que la población, en el marco de la responsabilidad social empresarial, demanda cada vez más un mayor aporte de las empresas hacia la sociedad, un mayor compromiso y evidencia de estas contribuciones, que generalmente se realizan a través de proyectos de desarrollo social ejecutados en las comunidades.

La presente investigación busca brindar un estudio sobre los indicadores de Responsabilidad Social para un 
adecuado control de proyectos de RSE financiados por empresas mineras, promoviendo la inversión privada efectiva en este tipo de proyectos, lo que permitirá complementar los insuficientes recursos públicos, mejorar la gestión de los mismos y llegar a un mayor número de localidades, delimitando qué criterios e indicadores son relevantes para empresas del sector minero, para esto se usará una metodología divida en tres etapas: (1) Identificación de los principales indicadores mostrados en proyectos mineros; (2) Revisión por expertos de los indicadores de Responsabilidad social identificados e identificación de criterios relevantes en minería; y (3) Identificación y diseño de los indicadores de Responsabilidad Social Empresarial.

\section{Responsabilidad social, rentabilidad social e indicadores}

\section{La Responsabilidad Social} Empresarial se ha convertido en una obligación social trascendental en diversos campos empresariales (Wang, Dou, y Jia 2016). Se ha investigado las influencias de la RSE en la gestión y el rendimiento corporativo en varios escenarios empresariales observando experiencias positivas en su implementación (Park, 2019). Desde la perspectiva de la gestión, las actividades de RSE son un deber importante para la supervivencia estratégica y sostenible de las empresas, la RSE se describe como la identificación, medición, monitoreo y reporte de los efectos sociales y económicos de una organización en la sociedad (Oyewumi et al, 2018).

La rentabilidad social, en muchos casos, es entendida como las adecuadas relaciones con los grupos de interés, mejor reputación e imagen, reconocimiento de las comunidades donde mantienen operaciones, motivación de sus trabajadores, entre los principales beneficios. En torno a estos conceptos surgen otros conceptos como el de marketing social, definido como un proceso en el cual una empresa adopta sus decisiones de marketing con un triple objetivo: interés empresarial, satisfacción del consumidor y el bienestar a largo plazo de éste y de la sociedad en su conjunto (Park, 2019). A su vez, Margolis en un trabajo de sistematización de un total de 251 artículos, muestra que existe una relación ligeramente positiva en los conceptos de rentabilidad y RSE (Margolis et al, 2009).

La medición de la responsabilidad social, es un proceso complejo y radica en cómo rentabilizar los resultados de la medición, es decir, cómo capitalizar para la empresa este aporte social, en especial el aporte externo que se realiza a través de diferentes estrategias $y$ acciones que implementa la empresa. En general, bajo el enfoque de gestión por resultados, los indicadores juegan un rol clave, resumen la información necesaria para efectuar la comparación entre lo planeado y lo ejecutado y hacen que esta comparación sea objetiva. Luego de haber fijado los objetivos y acciones necesarias para lograr los resultados deseados, el siguiente paso consiste en formular o seleccionar los indicadores relevantes y establecer las metas correspondientes a dichos resultados (Cracolici et al, 2010); por lo tanto, el no definir adecuadamente estos indicadores podría disminuir el interés de las empresas -no solo del sector minero-, para el financiamiento de nuevos proyectos, ya que al no contar con información adecuada, generaría 
Rojas de la Puente, Edward Enrique; Fanny Lucila Rimarachín Chavez; Oblitas Cruz, Jimy y Castro Silupu, Wilson

cierta incertidumbre llegando a repercutir incluso en el desarrollo de la sociedad (Cho, et al, 2019; Raut et al, 2019).

Se está viviendo en una época de inversión global, donde los proyectos mineros son ampliamente partícipes y donde la ejecución a gran escala es algo que se ha convertido en foco de desarrollo para las regiones donde se generan (Aarseth et al, 2017), por lo que este tipo de proyectos exigen cada vez más consideraciones sobre diversos problemas económicos, ambientales y sociales (Xue et al, 2015).

En este marco los reportes sobre RSE no solo han de conciliar la información social, medioambiental y económica, sino que también afronta el desafío de informar a los grupos de interés sobre las cuestiones que éstos requieren, siendo importante definir claramente qué indicadores se deberían reportar en los proyectos de RSE implementados por las empresas. Sorprendentemente, en la literatura de RSE hay una escasez de estudios empíricos que exploren este punto, tal como menciona Joo (2019), es necesario comprender mejor las dimensiones con respecto a los esfuerzos de RSE de una organización.

En Latinoamérica existe muy poca información de indicadores de RSE, un acercamiento sobre este tipo de trabajos dado por Beckman (2009), en Chile, muestra la capacidad de gestionar las relaciones con múltiples partes interesadas y las percepciones de autenticidad también fueron fundamentales para el éxito de la RSE.

Una parte importante de abordar las brechas en indicadores sociales, es tener en cuenta criterios como el compromiso, que se define como el grado en que las partes interesadas perciben a la organización como dedicada o constante en las iniciativas de RSE, en lugar de ajustar las iniciativas para satisfacer las tendencias actuales. En este punto Godfrey (2005) afirmó que, los compromisos de RSE consistentes de una empresa, son un indicador importante para evitar percepciones equivocadas de los esfuerzos de RSE de la compañía, además de mostrar el compromiso a largo plazo de la operación empresarial.

Otro criterio importante es la confiabilidad, que se define como el grado en que las partes interesadas perciben el programa de RSE y que realmente hace lo que promete hacer (Alhouti et al, 2016). Asimismo, se abordará en la investigación la relación con la comunidad, la que se define como el grado en que las partes interesadas perciben las iniciativas de RSE. De acuerdo a Mazutis y Slawinski (2015), estas iniciativas se perciben como más auténticas cuando sirven a los intereses de las partes interesadas y/o benefician a la comunidad local y apoyan a las personas dentro de la comunidad

\section{Identificación de indicadores de RSE en proyectos mineros}

Con la finalidad de identificar y evaluar las iniciativas de RSE se evaluaron tres proyectos mineros en Perú, ejecutados por empresas mineras multinacionales de tipo aurífero; $y$, por razones de confidencialidad no se hará referencia a la razón social de estas empresas.

La recolección de datos para el estudio, estuvo centrada en la aplicación de una entrevista al encargado del área de Responsabilidad Social o su equivalente, dentro de la empresa y el análisis de documentación de los proyectos ejecutados como parte de sus programas de Responsabilidad Social Empresarial con las comunidades, se analizaron para la investigación un total 
de ocho proyectos de responsabilidad social ejecutados. Con el propósito de identificar los indicadores relevantes de RSE financiados con fondos de empresas mineras en el ámbito de impacto de sus proyectos, se propone la siguiente metodología: Etapa (1) Identificación de los principales indicadores mostrados en proyectos ejecutados en el rango de tiempo 2008-2017, Etapa (2) Revisión por expertos de los indicadores de RSE identificados en la fase anterior e identificación de criterios relevantes para proyectos de RSE en minería; y Etapa (3) Identificación y diseño de los indicadores de RSE.

\section{Etapa 1: Identificación de indicadores de RSE en ocho proyectos mineros financiados}

La primera fase consistió en identificar los indicadores de RSE propuestos en ocho proyectos de Responsabilidad Social financiados, dos (02) en las líneas de educación y seis (6) en la línea de desarrollo de capacidades productivas, los cuales cuentan con indicadores y en su totalidad ascienden a una inversión de 6 millones de dólares realizada por las empresas mineras entre el periodo 2008 y 2017.

Luego del análisis documental, se observa que las oficinas de Responsabilidad Social o sus equivalentes en las empresas mineras, son los que miden los indicadores, éstos fueron planteados por el mismo equipo de ejecución del proyecto, así, en la Tabla 1 se muestran los indicadores que cada proyecto evaluó, donde se observa que se centran en la medición del desarrollo de las actividades y avance de metas de cada proyecto, mas no miden conceptos de impacto social.

Tabla 1

Indicadores de los proyectos evaluados

\begin{tabular}{|c|c|c|c|}
\hline $\begin{array}{l}\text { Tipo de } \\
\text { Proyecto }\end{array}$ & Objetivo & Beneficiarios & Indicadores \\
\hline \multirow[t]{2}{*}{$E^{*}$} & \multirow{2}{*}{$\begin{array}{l}\text { Desarrollar competen- } \\
\text { cias }\end{array}$} & \multirow[t]{2}{*}{11292} & $\begin{array}{l}\% \text { de estudiantes de las institucio- } \\
\text { nes beneficiadas }\end{array}$ \\
\hline & & & $\mathrm{N}^{\circ}$ de docentes certificados \\
\hline \multirow{4}{*}{$E$} & \multirow{4}{*}{$\begin{array}{l}\text { Mejorar los niveles de } \\
\text { aprendizaje }\end{array}$} & \multirow{4}{*}{4858} & $\begin{array}{l}\% \text { de reducción de la deserción } \\
\text { escolar }\end{array}$ \\
\hline & & & $\%$ de la repitencia escolar \\
\hline & & & $\begin{array}{l}\% \text { de los estudiantes han mejora- } \\
\text { do sus capacidades en comuni- } \\
\text { cación. }\end{array}$ \\
\hline & & & $\begin{array}{l}\% \text { Estudiantes han desarrollado } \\
\text { sus capacidades en matemáticas }\end{array}$ \\
\hline
\end{tabular}


Control de proyectos de Responsabilidad Social empresarial: Estudio en empresas mineras

Rojas de la Puente, Edward Enrique; Fanny Lucila Rimarachín Chavez; Oblitas Cruz, Jimy y Castro Silupu, Wilson

\begin{tabular}{|c|c|c|c|}
\hline $\mathrm{DCP}^{* *}$ & $\begin{array}{l}\text { Mejorar la calidad, } \\
\text { productividad y compe- } \\
\text { titividad }\end{array}$ & 600 & $\begin{array}{l}\% \text { de incremento de ingresos } \\
\% \text { de incremento en la producti- } \\
\text { vidad }\end{array}$ \\
\hline DCP & $\begin{array}{l}\text { Mejorar la calidad, } \\
\text { productividad y compe- } \\
\text { titividad }\end{array}$ & 200 & $\begin{array}{l}\mathrm{N}^{\circ} \text { de jóvenes han culminaron } \\
\text { con éxito el programa de capaci- } \\
\text { tación. } \\
\mathrm{N}^{\circ} \text { de jóvenes capacitados por el } \\
\text { proyecto han accedido a trabajo } \\
\text { formal. }\end{array}$ \\
\hline \multirow[t]{2}{*}{ DCP } & \multirow[t]{2}{*}{$\begin{array}{l}\text { Mejorar el aprovecha- } \\
\text { miento de alimentos de } \\
\text { la zona de impacto }\end{array}$} & \multirow[t]{2}{*}{130} & $\begin{array}{l}\% \text { Incremento de ingresos econó- } \\
\text { micos. } \\
\mathrm{N}^{\circ} \text { de empleos incrementales al } \\
\text { termino del proyecto. }\end{array}$ \\
\hline & & & Incremento de la producción \\
\hline DCP & $\begin{array}{l}\text { Incrementar los ingresos } \\
\text { económicos, a través } \\
\text { del fortalecimiento de } \\
\text { sus capacidades organi- } \\
\text { zacionales y financieras. }\end{array}$ & 4044 & Ratio Beneficio / Costo \\
\hline \multirow{3}{*}{ DCP } & \multirow{3}{*}{$\begin{array}{l}\text { Promover el desarrollo } \\
\text { de nuevos emprendi- } \\
\text { mientos }\end{array}$} & \multirow{3}{*}{350} & $\mathrm{~N}^{\circ}$ de jóvenes motivados \\
\hline & & & $\begin{array}{l}\mathrm{N} \text { de jóvenes capacitados } \\
\mathrm{N}^{\circ} \text { de jóvenes asesorados para la } \\
\text { elaboración y puesta en marcha } \\
\text { de planes de negocio. }\end{array}$ \\
\hline & & & $\begin{array}{l}N^{\circ} \text { de jóvenes presentaron planes } \\
\text { de negocio }\end{array}$ \\
\hline \multirow{3}{*}{$\mathrm{DCP}$} & \multirow{3}{*}{$\begin{array}{l}\text { Fortalecer el autoem- } \\
\text { pleo mediante el apoyo } \\
\text { a iniciativas de los jóve- } \\
\text { nes participantes }\end{array}$} & \multirow{3}{*}{200} & $\begin{array}{l}N^{\circ} \text { de jóvenes capacitados técni- } \\
\text { camente }\end{array}$ \\
\hline & & & $\mathrm{N}^{\circ}$ de negocios implementados \\
\hline & & & $\begin{array}{l}\mathrm{N}^{\circ} \text { de jóvenes graduados como } \\
\text { emprendedores }\end{array}$ \\
\hline
\end{tabular}

$\mathrm{E}^{*}=$ Educación $\quad \mathrm{DCP}^{* *}=$ Desarrollo de capacidades productivas

Fuente: elaboración propia

Este primer hallazgo, muestra que los proyectos solo ponen énfasis en los indicadores relacionados a temas operativos o ligados al resultado esperado, pero al no incluir indicadores de responsabilidad social, podría generar el no contar con evidencias formales del retorno social del proyecto, y que la empresas que desarrollan estos proyectos disminuyan gradualmente su interés de contribuir al desarrollo de las comunidades, distritos y región de 
influencia del proyecto minero; e, incluso el no contar con datos claros de retorno social, podría ocasionar la imposibilidad de informar adecuadamente a las comunidades generando dificultades para el normal funcionamiento o licencia social; a este tipo de conclusión, se llegó en otros estudios donde se usaron técnicas multicriterio para mejorar sus indicadores (González et al, 2015), además de ello, es imprescindible contar indicadores claros pues existen en la literatura relaciones positivas (Lee et al, 2017) y negativas (Testa y D'Amato, 2017) entre RSE y el indicador medido.

Como parte de la investigación, se realizó un estudio descriptivo a través de 520 encuestas distribuidas entre los beneficiarios de los proyectos de RSE, evaluados con el objetivo de conocer dos puntos importantes: "conoce la institución que trabajó RSE en su comunidad" (Gráfico 1-a); y, "considera que el financiador contribuye a su comunidad con el proyecto" (gráfico 1-b).

\section{Gráfico 1 \\ Resultados de encuestas expresadas en porcentaje}

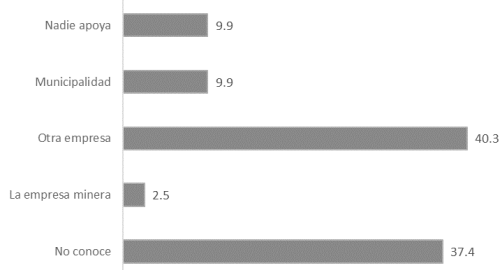

(a)

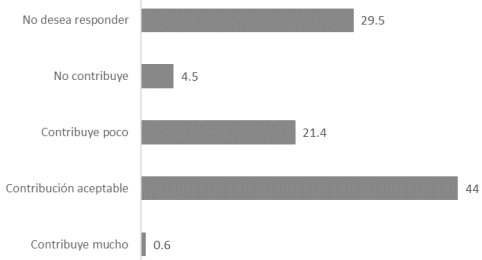

Fuente: elaboración propia

Al indagar en la población beneficiaria de los proyectos de RSE evaluados, acerca del conocimiento de la intervención de las empresas mineras, las respuestas evidencian que, el $37.4 \%$ manifiesta no conocer ninguna institución, el $40.3 \%$ identifica a otra empresa como la ejecutora del proyecto, en tanto que sólo el $2.5 \%$ sabe que la empresa minera trabaja en su comunidad. Finalmente, al indagar si la población beneficiaria del proyecto considera que la empresa minera financiadora contribuye a su comunidad, se encontró que el $44 \%$ considera un aporte aceptable, aunque en diversa medida; y, solo el $4.5 \%$ considera que no contribuye.

\section{Etapa 2: Identificación de criterios de RSE en minería por panel de expertos}

Con la finalidad de analizar los indicadores identificados en la Tabla 1 , se convocó a un grupo de nueve expertos nacionales en proyectos de responsabilidad social en industrias extractivas. Los expertos seleccionados para la presente investigación contaron 
con el siguiente perfil: más de 5 años de experiencia en proyectos de desarrollo, haber participado en el diseño o ejecución de proyectos de Responsabilidad Social, haber trabajado para empresas que cofinancien los proyectos. Los expertos valoraron la importancia de los indicadores resultantes de la fase anterior y propusieron indicadores relacionados a responsabilidad social que podrían ser más factibles en el análisis de este tipo de proyectos.

Se muestra en el cuadro 1, los indicadores sistematizados por el grupo de expertos, que son de interés para las empresas del sector minero financiador y que puedan servir de base para elaborar una propuesta de indicadores de rentabilidad social.

\section{Cuadro 1 \\ Sistematización de indicadores de responsabilidad social de interés para empresas mineras}

\begin{tabular}{|c|c|c|}
\hline Criterio & Concepto & Indicadores usados \\
\hline Inversión Social & $\begin{array}{l}\text { Asignación de recursos a poblacio- } \\
\text { nes del ámbito de influencia de la } \\
\text { empresa. }\end{array}$ & $\begin{array}{l}\text { Inversión en proyectos } \\
\text { Inversión directa a requerimiento de la comu- } \\
\text { nidad } \\
\text { Beneficio social } \\
\text { Beneficio económico }\end{array}$ \\
\hline Ámbito & $\begin{array}{l}\text { Extensión geográfica o política que } \\
\text { es prioridad de la empresa. }\end{array}$ & $\begin{array}{l}\text { Limites políticos } \\
\text { Limites de Cuencas } \\
\text { Limite cultural }\end{array}$ \\
\hline $\begin{array}{l}\text { Prioridades de los proyectos de } \\
\text { desarrollo }\end{array}$ & $\begin{array}{l}\text { Conjunto de criterios que tiene en } \\
\text { cuenta la empresa al momento de } \\
\text { diseñar los proyecto. }\end{array}$ & $\begin{array}{l}\text { Alineamiento a los Objetivos de Desarrollo } \\
\text { Sostenible } \\
\text { Planes de desarrollo local o regional } \\
\text { Nivel de pobreza }\end{array}$ \\
\hline Temática de proyectos & $\begin{array}{l}\text { Aspecto principal para la mejora de } \\
\text { la calidad de vida que propone el } \\
\text { proyecto }\end{array}$ & $\begin{array}{l}\text { Salud } \\
\text { Educación } \\
\text { Productivo } \\
\text { Agua }\end{array}$ \\
\hline $\begin{array}{l}\text { Instrumentos para identificar las } \\
\text { problemáticas }\end{array}$ & $\begin{array}{l}\text { Conjunto de herramientas que permi- } \\
\text { ten realizar el proceso de identifica- } \\
\text { ción de la problemática. }\end{array}$ & $\begin{array}{l}\text { Diagnósticos participativos } \\
\text { Talleres } \\
\text { Dialogo con las comunidades } \\
\text { Mapeo de grupo de interés }\end{array}$ \\
\hline Alcance del proyecto & $\begin{array}{l}\text { Metas que se establecen en el pro- } \\
\text { yecto de acuerdo a su propósito }\end{array}$ & $\begin{array}{l}\text { Impacto } \\
\text { Costo/ Beneficio } \\
\text { Costo / Efectividad }\end{array}$ \\
\hline Herramientas de medición & $\begin{array}{l}\text { Conjunto de instrumentos que permi- } \\
\text { ten mensurar los cambios que se dan } \\
\text { por la contribución de la empresa }\end{array}$ & $\begin{array}{l}\text { M\&E } \\
\text { Seguimiento } \\
\text { Taller participativo } \\
\text { Apalancamiento }\end{array}$ \\
\hline $\begin{array}{l}\text { Percepción hacia la empresa } \\
\text { minera }\end{array}$ & $\begin{array}{l}\text { Juicios verdaderos o falsos de los } \\
\text { grupos de interés con relación a la } \\
\text { empresa. }\end{array}$ & $\begin{array}{l}\text { Credibilidad } \\
\text { Equilibrio de intereses } \\
\text { Articulación }\end{array}$ \\
\hline Imagen & $\begin{array}{l}\text { Como se percibe una empresa o } \\
\text { institución en función a algunas de } \\
\text { sus características }\end{array}$ & $\begin{array}{l}\text { Actitud de los trabajadores } \\
\text { Interculturalidad } \\
\text { Comunicación oportuna } \\
\text { Respeto al medio ambiente }\end{array}$ \\
\hline Confianza & $\begin{array}{l}\text { Nivel de seguridad o esperanza que } \\
\text { tienen las comunidades del entorno } \\
\text { de una empresa hacia sus acciones }\end{array}$ & $\begin{array}{l}\text { Aceptación } \\
\text { Presencia } \\
\text { Respeto } \\
\text { Sostenibilidad }\end{array}$ \\
\hline
\end{tabular}


En estudios en Perú, los indicadores priorizaron temas en desarrollo sostenible, confluye hacia tres ejes: económico, social y ambiental (Barbachan, 2017), todo ello con el objetivo de mejorar la satisfacción del consumidor. En el estudio de Hasenpusch (2018), muestra a través del análisis de la norma ISO 26000 que, la RSE se puede medir en términos de inversión social (apoyo comunitario), alcance y medición del proyecto (evaluación de desempeño), e imagen; éstos concuerdan con indicadores planteados en investigaciones latinoamericanas (González et al, 2015) e internacionales (Cho et al, 2019; Hou, 2019; Kubareva et al, 2018).

Cabe resaltar que, los expertos que analizaron los indicadores de los proyectos mineros en esta investigación, han priorizado y realizado una aproximación al concepto y a los aspectos principales que cada criterio contempla. Algunas apreciaciones finales expresadas por el grupo de expertos se detallan a continuación: (a) los mecanismos más recurrentes para la canalización de la inversión social son en el corto plazo, generalmente, a través del área de relaciones sociales o comunitarias de las empresas mineras; mientras que a largo plazo, la canalización de la inversión más efectiva, es a través de proyectos de desarrollo; (b) se observa que las empresas mineras que invierten en programas y proyectos tienen en cuenta dos aspectos: las necesidades de la población del ámbito de influencia y la continuidad operativa del proyecto minero o extractivo; (c) los mecanismos más recurrentes para determinar la problemática de las comunidades son los diagnósticos, así como los estudios de impacto ambiental; (d) para el diseño de proyectos solo se tiene en cuenta el ámbito de influencia más no el criterio del impacto en el desarrollo; (e) no se cuenta con herramientas de medición por el área de relaciones sociales o comunitarias; y, (f) el tema de reputación es un tema altamente sensible, tal es así que, los pobladores tienen mayor propensión a recordar lo negativo de los proyectos.

\section{Etapa 3: Identificación de Indicadores relevantes de los proyectos de RSE en minería}

De las reuniones con expertos y a través del uso de entrevistas abiertas, se identificó un paquete de indicadores que permita recoger características relevantes de los proyectos, luego se aplicó un cuestionario y se sistematizó a través de una matriz de priorización, teniendo en cuenta su definición, rol, tipología y evaluabilidad. Este proceso siguió la secuencia mostrada en el diagrama 1. Bajo este análisis se seleccionaron los siguientes criterios: (a) inversión social, (b) imagen y confianza, (c) prioridades de los proyectos de desarrollo, (d) apalancamiento de recursos - aliados; y, (e) rentabilidad social. Este estudio adopta el valor $\alpha$ de Cronbach como una herramienta para el nivel de confiabilidad, encontrándose que los valores en cada criterio fueron superiores a 0.93 , por lo que la consistencia interna de todos los criterios es bastante buena. 


\section{Diagrama 1 \\ Secuencia de trabajo ejecutada por los expertos para definir crite- rios e indicadores relevantes en proyectos mineros}

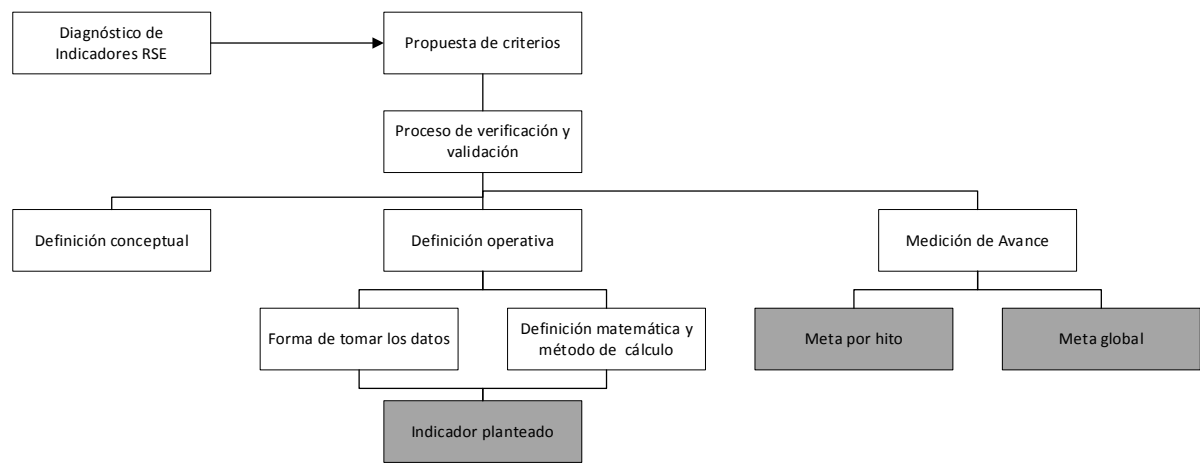

Fuente: elaboración propia

Para cada uno de los cinco (05) criterios priorizados, se plantearon y seleccionaron indicadores en función a la experiencia en proyectos desde el enfoque de responsabilidad social de todos los expertos; además, se tuvo en cuenta los siguientes parámetros para el diseño de indicadores: definición precisa, identificable y sencillo, flexible, sectorial, evolutivo, único e informativamente completo (González et al, 2015). Los indicadores propuestos se muestran en la diagrama 2, relacionados al criterio priorizado, al cual contribuiría su incorporación en los proyectos de desarrollo a ser financiados por las empresas del sector minero; además, se detallan en el cuadro 2, donde se observa su respectiva definición y método de cálculo.

A partir de estos criterios, se ha elaborado una propuesta inicial de indicadores que sirva de herramienta a las empresas mineras para los procesos de diseño y evaluación de proyectos de desarrollo.
Cabe mencionar que, si bien, se podrían elaborar otros indicadores, los que se presentan son los que han sido priorizados desde la experiencia de los expertos que han participado en la presente investigación. En este sentido, González (2015) en su investigación sobre identificación de indicadores relevantes del desempeño de la responsabilidad social empresarial, señala entre sus conclusiones que "...a pesar de la proliferación de información de RSE, existe la necesidad de sintetizar esa información en un número de indicadores manejable, que siendo relevantes desde el punto de vista técnico, lo sea también desde el punto de vista de su legitimación social."

Es importante mencionar que, las empresas mineras podrían incorporar estos indicadores de manera directa en el diseño de los proyectos de RSE, o considerarlos en términos de referencia cuando encarguen el diseño o evaluación de un proyecto; e, incluso discernir entre un conjunto de proyectos 


\section{Diagrama 2 \\ Relación del criterio y el indicador planteado}

CRITERIO
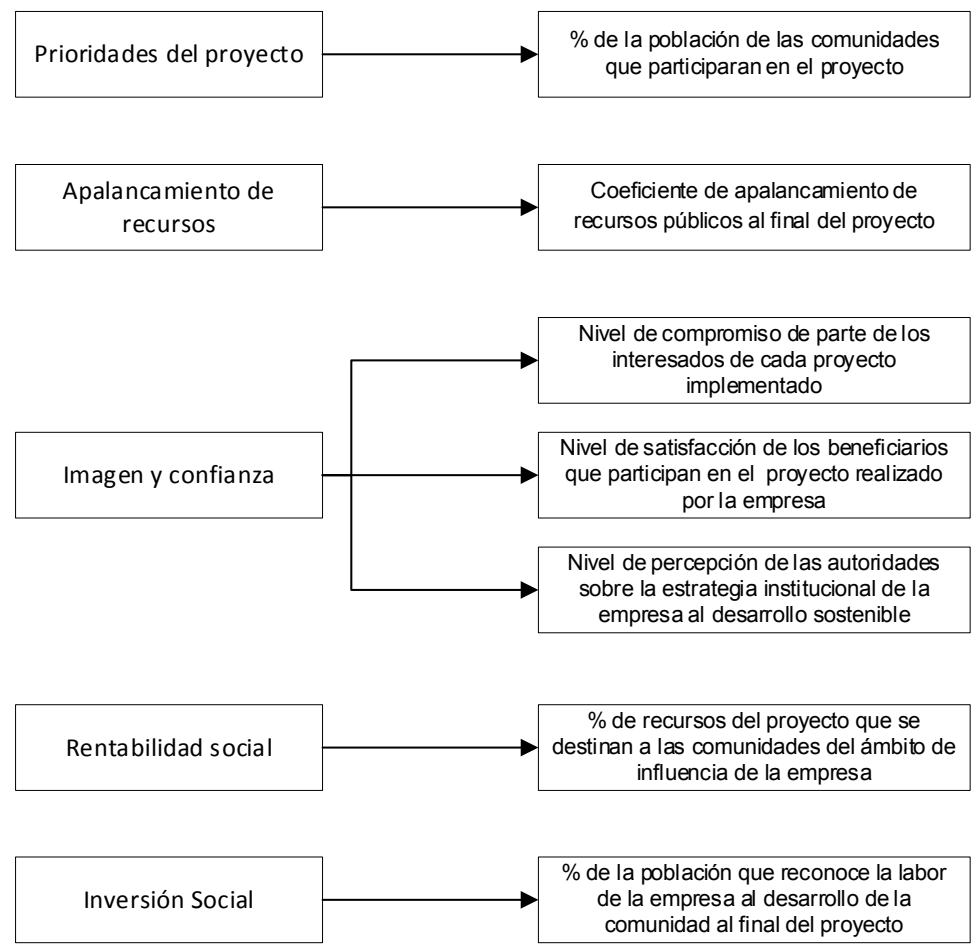

Fuente: elaboración propia

para su financiamiento al plantearse la interrogante ¿cuál proyecto es el que les podría generar mayor retorno social?.

Documentos que tratan de evaluar la efectividad de la inversión en RSE y sus implicaciones para la rentabilidad empresarial existen, pero no muestran el uso de indicadores sociales (Mukherjee et al, 2018), otras investigaciones como la de Borghesi, incluyen algunas variables de control corporativo a modo de indicadores, muchos de estos indicadores no son homogéneos, lo cual dificulta la comparación entre los resultados de cada intervención de proyectos de Responsabilidad Social.

Otro problema en los indicadores tradicionales lo plantea Kao (2018), donde manifiesta que es necesario controlar el problema de endogeneidad en la estimación de la relación entre el desempeño y la RSE, problema que 
encontró debido a un mal planteamiento de indicadores de medición de RSE, por lo que la propuesta de esta investigación cubre el vacío de tener indicadores adecuados y válidos.

El uso de expertos en el trabajo nos ayudó a tener una concepción clara de qué medir en este tipo de proyectos, ya que como se menciona, solo se venía midiendo indicadores de desarrollo del proyecto, mas no de índole de RSE. Otras investigaciones que usaron la generación de indicadores a través de expertos, manifiestan que las herramientas generadas son útiles y significativas, además de sencillas de usar y ayudan a comprender la relación entre la empresa y beneficiarios (Chen et al, 2018; Yu y Chen, 2014).

\section{Cuadro 2}

\section{Propuesta de indicadores según criterio}

\begin{tabular}{|c|c|c|}
\hline Propuesta de indicadores & Definición & Método de cálculo \\
\hline $\begin{array}{c}\text { \% de recursos del proyecto que se } \\
\text { destinan a las comunidades } \\
\text { del ámbito de influencia de la } \\
\text { empresa }\end{array}$ & $\begin{array}{c}\text { Asignación de presupuesto del proyecto que se } \\
\text { estima de manera directa a la implemen- } \\
\text { tación de las actividades que involucra a } \\
\text { la población. No se consideran los gastos } \\
\text { administrativos y operativos del proyecto. }\end{array}$ & $\begin{array}{c}\text { ((Inversión en componentes del } \\
\text { proyecto) / (Presupuesto } \\
\text { Total del Proyecto) }) * 100\end{array}$ \\
\hline $\begin{array}{l}\text { Coeficiente de apalancamiento de } \\
\text { recursos públicos al final del } \\
\text { proyecto }\end{array}$ & $\begin{array}{l}\text { Se refiere al nivel de movilización de recursos } \\
\text { económicos y no económicos que con- } \\
\text { sidera el diseño del proyecto de otros } \\
\text { actores públicos y privados. }\end{array}$ & $\begin{array}{l}\text { Presupuesto financiado por la } \\
\text { empresa / Presupuesto fi- } \\
\text { nanciado x otras empresas } \\
\text { privadas o pública }\end{array}$ \\
\hline $\begin{array}{c}\% \text { de la población que reconoce la } \\
\text { labor de la empresa al desa- } \\
\text { rrollo al final del proyecto }\end{array}$ & $\begin{array}{c}\text { Nivel de conocimiento de la población objetivo } \\
\text { del proyecto, así como de los grupos de } \\
\text { interés sobre el rol que cumple la empresa } \\
\text { en el proceso de ejecución del proyecto. }\end{array}$ & $\begin{array}{c}\left(\left(\mathrm{N}^{\circ} \text { de personas que conocen }\right.\right. \\
\text { el trabajo de la empresa }) / \\
(\text { Población })) * 100\end{array}$ \\
\hline $\begin{array}{c}\text { Nivel de compromiso de parte } \\
\text { de los interesados de cada } \\
\text { proyecto implementado }\end{array}$ & $\begin{array}{l}\text { Se refiere al grado de involucramiento de los } \\
\text { diferentes interesados en los procesos de } \\
\text { implementación del proyecto. }\end{array}$ & $\begin{array}{c}\mathrm{N}^{\circ} \text { de participaciones de los } \\
\text { grupos de interés en los } \\
\text { espacios que promueve } \\
\text { el proyecto durante su } \\
\text { ejecución. }\end{array}$ \\
\hline $\begin{array}{l}\text { Nivel de satisfacción de los } \\
\text { beneficiarios que participan } \\
\text { en el proyecto realizado por } \\
\text { la empresa }\end{array}$ & $\begin{array}{l}\text { Se refiere a la conformidad de la población } \\
\text { objetivo con relación a los cambios alcan- } \\
\text { zados por participar en el proyecto. }\end{array}$ & $\begin{array}{c}\mathrm{N}^{\circ} \text { de testimonios de los partici- } \\
\text { pantes del proyecto a favor } \\
\text { de la empresa. }\end{array}$ \\
\hline
\end{tabular}

Fuente: Elaboración propia 
Los indicadores planteados, tienen una mayor profundidad en la información que recoge de los proyectos de RSE en minería, lo cual permite evaluar correctamente la gestión llevada. Además, al tener básicamente un enfoque cuantitativo conduce al relevamiento sistemático de datos, generando comparaciones anuales para cruzar con otros datos relevantes para la empresa, además de generar un control mayor a través de auditorias y monitoreos de metas en materia de RSE. De manera integrada con toda la empresa, este tipo de control permitirá hacer más consistente la planificación futura de las empresas mineras, consolidando directivas para el establecimiento adecuado de objetivos y metas en RSE en minería.

Por último, en base a los resultados obtenidos se puede afirmar que, el planteamiento de estos indicadores relaciona dos conceptos: la responsabilidad social empresarial y el desarrollo sostenible de los proyectos, ya que su aplicación adecuada puede reducir costos y proporcionar valor a largo plazo. Sin embargo, aún existe un amplio debate sobre el concepto de RSE con un enfoque en temas sociales, sobre todo la falta de índices adecuados para establecer el desempeño de la organización en términos de sostenibilidad (Elmualim, 2017); motivo por el cual, creemos firmemente que la propuesta será un aporte significativo para demostrar el buen desempeño social a través de proyectos de RSE, otros estudios han planteado motivos similares en temas medioambientales logrando resultados importantes (Chollet y Sandwidi, 2018).

En un estudio planteado por Vartiak (2016), se muestra como principal hallazgo que en América,
Europa y Asia Pacífico, el porcentaje de empresas con informes de RSE son similares en su estructura, mas no lo son sus indicadores, por lo que nuestra propuesta ayudará también a unificar estos criterios a fin de encontrar un punto común de discusión futura de los impactos generados de los proyectos de Responsabilidad Social Empresarial.

\section{Reflexiones finales}

Se espera que la investigación presentada sea un punto de discusión y de referencia, a fin de ayudar a enfocar indicadores adecuados para los futuros proyectos de responsabilidad social empresarial, dentro del rubro de empresas mineras. La inversión minera como de otros sectores, en proyectos de RSE, debe generar un valor compartido para la empresa, comunidad y Estado, la cual debe ser mensurable desde que se gesta el proyecto para garantizar su efectividad.

Se ha logrado elaborar una propuesta de indicadores que sirve de herramienta y contribuye a generar rentabilidad social para las empresas financiadoras mineras, desde el proceso de diseño y evaluación de este tipo de proyectos. Los criterios identificados pueden considerarse un punto de partida para la generación de indicadores ad hoc en otros sectores empresariales, que contemplen un enfoque de responsabilidad social y desarrollo sostenible.

Es necesario implementar indicadores de responsabilidad social en los proyectos que financian las empresas del sector minero; de tal manera que, permita medir su retorno en términos de imagen y confianza como una estrategia que responda a ambos intereses: empresa y comunidad; y, que 
Control de proyectos de Responsabilidad Social empresarial: Estudio en empresas mineras

Rojas de la Puente, Edward Enrique; Fanny Lucila Rimarachín Chavez; Oblitas Cruz, Jimy y Castro Silupu, Wilson

motive a las empresas a incrementar esta contribución.

\section{Referencias Bibliográficas}

Aarseth, Wenche, Tuomas Ahola, Kirsi Aaltonen, Andreas Økland, y Bjørn Andersen (2017), «Project sustainability strategies: A systematic literature review». International Journal of Project Management 35(6):107183.

Alhouti, Sarah, Catherine M. Johnson, y Betsy Bugg Holloway (2016), "Corporate social responsibility authenticity: Investigating its antecedents and outcomes». Journal of Business Research 69(3):1242-49.

Amos, G. J. (2018), «Corporate social responsibility in the mining industry: an exploration of host-communities' perceptions and expectations in a developing-country». Corporate Governance (Bingley) 18(6):1177-95.

Barbachan, Madeleine (2017), «La Responsabilidad Social Empresarial en el Perú: Desafíos y Oportunidades». InnovaG 0(2):56-62.

Beckman, Terry, Alison Colwell, y Peggy H. Cunningham (2009), «The Emergence of Corporate Social Responsibility in Chile: The Importance of Authenticity and Social Networks». Journal of Business Ethics 86(2):191.

Borghesi, Richard, Joel F. Houston, y Andy Naranjo (2014), «Corporate socially responsible investments: CEO altruism, reputation, and shareholder interests». Journal of Corporate Finance $26: 164-81$.

Chen, Chun-Shuo, Chih-Ching Yu, y JerSan Hu (2018), "Constructing performance measurement indicators to suggested corporate environmenta responsibility framework». Technological Forecasting and Social Change 135:33-43.

Cho, S. J., C. Y. Chung, y J. Young (2019), "Study on the relationship between CSR and financial performance». Sustainability (Switzerland) 11(2).

Chollet, Pierre y Blaise W. Sandwidi (2018), «CSR engagement and financial risk: A virtuous circle? International evidence». Global Finance Journal 38:65-81.

Combs, Alan, Daria Varenova, y Martin Samy (2013), "Corporate social responsibility and profitability: trade $\square$ off or synergy: Perceptions of executives of FTSE All $\square$ Share companies». Sustainability Accounting, Management and Policy Journal 4(2):190-215.

Cracolici, Maria Francesca, Miranda Cuffaro, y Peter Nijkamp (2010), «The Measurement of Economic, Social and Environmental Performance of Countries: A Novel Approach». Social Indicators Research 95(2):33956.

Dunlap, A. (2019), «'Agro sí, mina NO!' the Tía Maria copper mine, state terrorism and social war by every means in the Tambo Valley, Peru». Political Geography 71:10-25.

Elmualim, Abbas (2017), «CSR and Sustainability in FM: Evolving Practices and an Integrated Index». Procedia Engineering 180:1577-84.

Frederiksen, Tomas (2018) «Corporate social responsibility, risk and development in the mining industry». Resources Policy 59:495-505.

Galant, Adriana y Simon Cadez (2017), «Corporate social responsibility and financial performance relationship: a review of measurement approach- 
es». Economic Research-Ekonomska Istraživanja 30(1):676-93.

Godfrey, Paul C. (2005), «The Relationship Between Corporate Philanthropy And Shareholder Wealth: A Risk Management Perspective». Academy of Management Review 30(4):777-98.

González, Marta de la Cuesta, Eva Pardo Herrasti, y Juan Diego Paredes Gázquez (2015), «Identificación de indicadores relevantes del desempeño RSE mediante la utilización de técnicas multicriterio». Innovar 25(55):75-88.

Hasenpusch, W. (2018), «The social responsibility of companies and the ISO 26000 (Part 1)». Galvanotechnik 109(7):1450-54. He, L., C. Wu, X. Yang, y J. Liu (2019), «Corporate social responsibility, green credit, and corporate performance: an empirical analysis based on the mining, power, and steel industries of China». Natural Hazards 95(1-2):73-89.

Hou, T. C. T. (2019), «The relationship between corporate social responsibility and sustainable financial performance: firm-level evidence from Taiwan». Corporate Social Responsibility and Environmental Management 26(1):19-28.

Hoyos, Carrillo y Sandra Verónica (2012), «Comunidades y minería : la comunicación en el conflicto». Pontificia Universidad Católica del Perú.

Joo, Soyoung, Elizabeth G. Miller, y Janet S. Fink (2019), "Consumer evaluations of CSR authenticity: Development and validation of $a$ multidimensional CSR authenticity scale». Journal of Business Research 98:236-49.

Kao, Erin H., Chih-Chuan Yeh, Li-Hsun Wang, y Hung-Gay Fung (2018), «The relationship between CSR and performance: Evidence in China». Pacific-Basin Finance Journal 51:155-70.

Kubareva, I., O. Maliarchuk, y N. Pohuda (2018), "Corporate social responsibility of Ukrainian tourist enterprises: Identity, strategy and performance». Eastern Journal of European Studies 9(2):145-67.

Lee, Chen-Ying, Wei-Chen Chang, y Hsin-Ching Lee (2017), «An investigation of the effects of corporate social responsibility on corporate reputation and customer loyalty - evidence from the Taiwan non-life insurance industry». Social Responsibility Journal 13(2):355-69.

Margolis, Joshua D., Hillary Anger Elfenbein, y James P. Walsh (2009), Does It Pay to Be Good...And Does It Matter? A Meta-Analysis of the Relationship between Corporate Social and Financial Performance. SSRN Scholarly Paper. ID 1866371. Rochester, NY: Social Science Research Network.

Mazutis, Daina D. y Natalie Slawinski (2015), «Reconnecting Business and Society: Perceptions of Authenticity in Corporate Social Responsibility». Journal of Business Ethics 131(1):137-50.

Morales, O., A. N. Kleit, y G. H. Rees (2018), «Mining and community relations in Peru: can agreement be reached?» Academia Revista Latinoamericana de Administracion 31(3):605-24.

Mukherjee, Abhishek, Ron Bird, y Geeta Duppati (2018), «Mandatory Corporate Social Responsibility: The Indian experience». Journal of Contemporary Accounting \& Economics 14(3):254-65.

Oyewumi, Obafemi R., Oluwabunmi A. Ogunmeru, y Collins S. Oboh (2018), 
Control de proyectos de Responsabilidad Social empresarial: Estudio en empresas mineras

Rojas de la Puente, Edward Enrique; Fanny Lucila Rimarachín Chavez; Oblitas Cruz, Jimy y Castro Silupu, Wilson

«Investment in corporate social responsibility, disclosure practices, and financial performance of banks in Nigeria». Future Business Journal 4(2):195-205.

Park, Eunil (2019), «Corporate social responsibility as a determinant of corporate reputation in the airline industry». Journal of Retailing and Consumer Services 47:215-21.

Raut, R. D., S. Luthra, B. E. Narkhede, S. K. Mangla, B. B. Gardas, y P. Priyadarshinee. (2019), «Examining the performance oriented indicators for implementing green management practices in the Indian agro sector». Journal of Cleaner Production 215:926-43.

Sícoli Pósleman, C. y J. M. Sallan (2019), «Social license to operate in the mining industry: the case of Peru». Impact Assessment and Project Appraisal.

Slack, Keith (2012), «Mission impossible?: Adopting a CSRbased business model for extractive industries in developing countries". Resources Policy 37(2):179-84.

Testa, Mario y Antonio D'Amato (2017), "Corporate environmental responsibility and financial performance: does bidirectional causality work? Empirical evidence from the manufacturing industry». Social Responsibility Journal 13(2):221-34.

Tuulentie, S., G. Halseth, A. Kietäväinen, L. Ryser, y J. Similä (2019), «Local community participation in mining in Finnish Lapland and Northern British Columbia, Canada - Practical applications of CSR and SLO». Resources Policy 61:99-107.

Vartiak, Lukas (2016), «CSR Reporting of Companies on a Global Scale». Procedia Economics and Finance 39:176-83.

Walker, J. y G. Sarkodie (2019), «Adult education as corporate social responsibility in Canadian mining companies: performing the good citizen for greater self-regulation». Compare 49(2):298-317.

Wang, Qian, Junsheng Dou, y Shenghua Jia (2016), «A Meta-Analytic Review of Corporate Social Responsibility and Corporate Financial Performance: The Moderating Effect of Contextual Factors». Business \& Society 55(8):1083-1121.

Xue, Xiaolong, Ruixue Zhang, Xiaoling Zhang, Rebecca Jing Yang, y Hong Li (2015), «Environmental and social challenges for urban subway construction: An empirical study in China». International Journal of Project Management 33(3):576-88.

$\mathrm{Yu}$, Chih-Ching y Chun-Shuo Chen (2014), «From the Actual Practice of Corporate Environmental Strategy to the Creation of a Suggested Framework of Corporate Environmental Responsibility». Environmental Engineering Science 31(2):61-70. 